\title{
Colloidal synthesis of $\mathrm{MoSe}_{2}$, WSe 2 and their hierarchical structures as bifunctional electrocatalysts
}

\author{
Rajeev Rai ${ }^{1}$, Bidushi Sarkar ${ }^{2}$, Ranit Ram $^{3}$ and N Ravishankar ${ }^{4}$ \\ ${ }^{1}$ Indian Institute of Science, Bangalore, Karnataka, India, ${ }^{2}$ Indian Institute of Science, Bangalore, United States, \\ ${ }^{3}$ Indian Institute of Science, Kharagpur, West Bengal, India, ${ }^{4}$ Indian Institute of Science, United States
}

Owing to the non-trivial physical and chemical properties, layered metal dichalcogenides (LMDs) have been exploited for various technological applications. ${ }^{1,2}$ Crystallographically, LMDs have metal atoms sandwiched between two layers of chalcogen (X-M-X covalent bonding) and the X-M-X layers are weakly bonded by van der Waals forces. This provides an opportunity to tailor their properties through heterostructure formation either by stacking different layers on top of each other or stitching two different layers in the same plane.

Recently LMDs have shown promising results as a non-precious alternative for the electrochemical water splitting reactions. ${ }^{3,4}$ Practically, for efficient water splitting reaction, electrocatalysts should be compatible with hydrogen evolution reaction (HER) and oxygen evolution reaction (OER) in the same medium. Owing to sluggish kinetics for water splitting reaction, OER in alkaline medium is a bottleneck in the process. Theoretical and experimental evidence suggest that edges of the LMDs such as MoS2, MoSe2 are catalytically more active than basal planes., Thus, LMDs as electrochemical catalysts with a large number of exposed edge sites are desired. Similarly, heterostructure engineering with enhanced edge sites can also provide an opportunity to alter the electronic properties and can be a promising strategy to enhance the water-splitting reaction via a synergistic effect.

To overcome the low-yield and substrate effect of various synthetic approaches such as CVD, PVD, exfoliation and restacking, facile low-cost, energy-efficient wet chemical methods have been designed. Wet-chemical methods allow for synthesizing substrate-free nanostructures with high-yield, which are desirable for catalysis and sensing applications. However, one-step synthesis of heterostructures through the wet-chemical process is still challenging and tend to form alloyed nanostructures when the precursors are mixed. Herein, using metal precursors with different reactivity towards chalcogen metal, we have designed a synthesis method for MoSe2, WSe2 and hierarchical heterostructure Mose2@WSe2 through one-step colloidal synthesis. As-synthesized nanostructures have flower morphology with large number of exposed edges, desired for electrocatalysis. As-synthesized nanostructures have been used for the complete water-splitting reaction in an alkaline medium. The overpotential at current density $10 \mathrm{~mA} / \mathrm{cm} 2$ for hydrogen evolution reaction and oxygen evolution reaction requires only $148 \mathrm{mV}$ and $30 \mathrm{mV}$ positive as compared to commercial $\mathrm{Pt} / \mathrm{C}$ and $\mathrm{RuO} 2$, respectively.

Figure 1 shows the microstructure of MoSe2 and WSe2. The low magnification bright-field TEM micrograph of MoSe2 and WSe2, as shown in figure 1(a and d), reveals flower morphology with a large number of exposed edges. The corresponding diffraction pattern is shown in figure 1(b and e). Owing to flower morphology, SAED pattern shows the polycrystalline nature. Figure 1(c and f) shows the high-resolution TEM (HRTEM) micrograph, which shows the formation of a few layered MoSe2 and WSe2.

Owing to the reactivity difference of the metal ion precursor (W and Mo) towards the chalcogen, our reaction scheme was extended to synthesize hierarchical heterostructures. Figure 2 shows the representative microstructural and elemental distribution of Mo, W, and Se. As-synthesized hierarchical heterostructures have flower morphology shown in low-mag bright-field TEM micrograph in figure 2(a). The corresponding SAED pattern shown in figure 2(b) shows polycrystalline nature due to flower morphology. HRTEM micrograph shown in figure 2(c) shows the (002) lattice plane with an extended spacing of $6.9 \AA$, which is more than the reported d-spacing of $6.49 \AA$ for $(002)$. However, due to negligible lattice mismatch between $\mathrm{MoSe} 2$ and WSe2, the distinction between alloy or 
hierarchical heterostructure through SAED and HRTEM micrograph is difficult. To check the distribution of Mo, $\mathrm{W}$ and Se, STEM-EDS mapping was performed. Figure 2(d) shows the HAADF-STEM micrograph and the corresponding elemental map of Mo, $\mathrm{W}$ and Se are shown in figure 2(e, $\mathrm{f}$ and $\mathrm{g}$ ). Se is uniformly distributed throughout the nanostructure; however, Mo and W show phase segregation. Mo being rich in the core, and W is predominantly present in the outer sheets.

Owing to the flower morphology with more number of exposed edges, as-synthesized nanostructures have been explored as catalyst for electrochemical HER and OER. Heterostructures show better electrochemical performance in comparison to MoSe2 and WSe2. The overpotential for heterostructures required to achieve $10 \mathrm{~mA} / \mathrm{cm} 2$ is only $148 \mathrm{mV}$ (HER) and $30 \mathrm{mV}$ (OER) with respect to $\mathrm{Pt} / \mathrm{C}$ and $\mathrm{RuO} 2$. The improved activity of heterostructures can be attributed to synergistic effect and better electron transfer at the coupled heterostructure as evident by reduced Tafel slope $(51 \mathrm{mV} / \mathrm{dec})$ for heterostructure. Hierarchical heterostructure also shows better stability after $20 \mathrm{~h}$ of the electrochemical cycle as compared to commercial $\mathrm{Pt} / \mathrm{C}$ and $\mathrm{RuO} 2$ with current retention of $87.9 \%$ in comparison to commercial couple $\mathrm{Pt} / \mathrm{C}$ and $\mathrm{RuO} 2$.
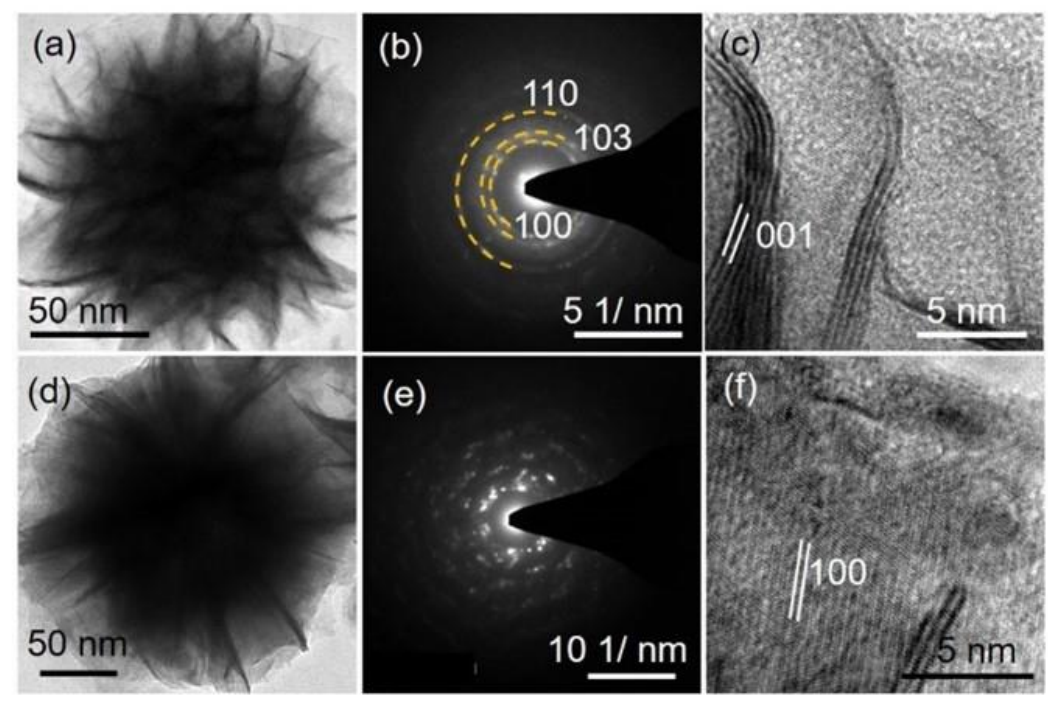

Figure 1. Bright-field TEM micrograph, selected area electron diffraction pattern and high-resolution TEM micrograph of $\operatorname{MoSe} 2(a, b$ and $c)$ and WSe2 (d, e and f) respectively. 

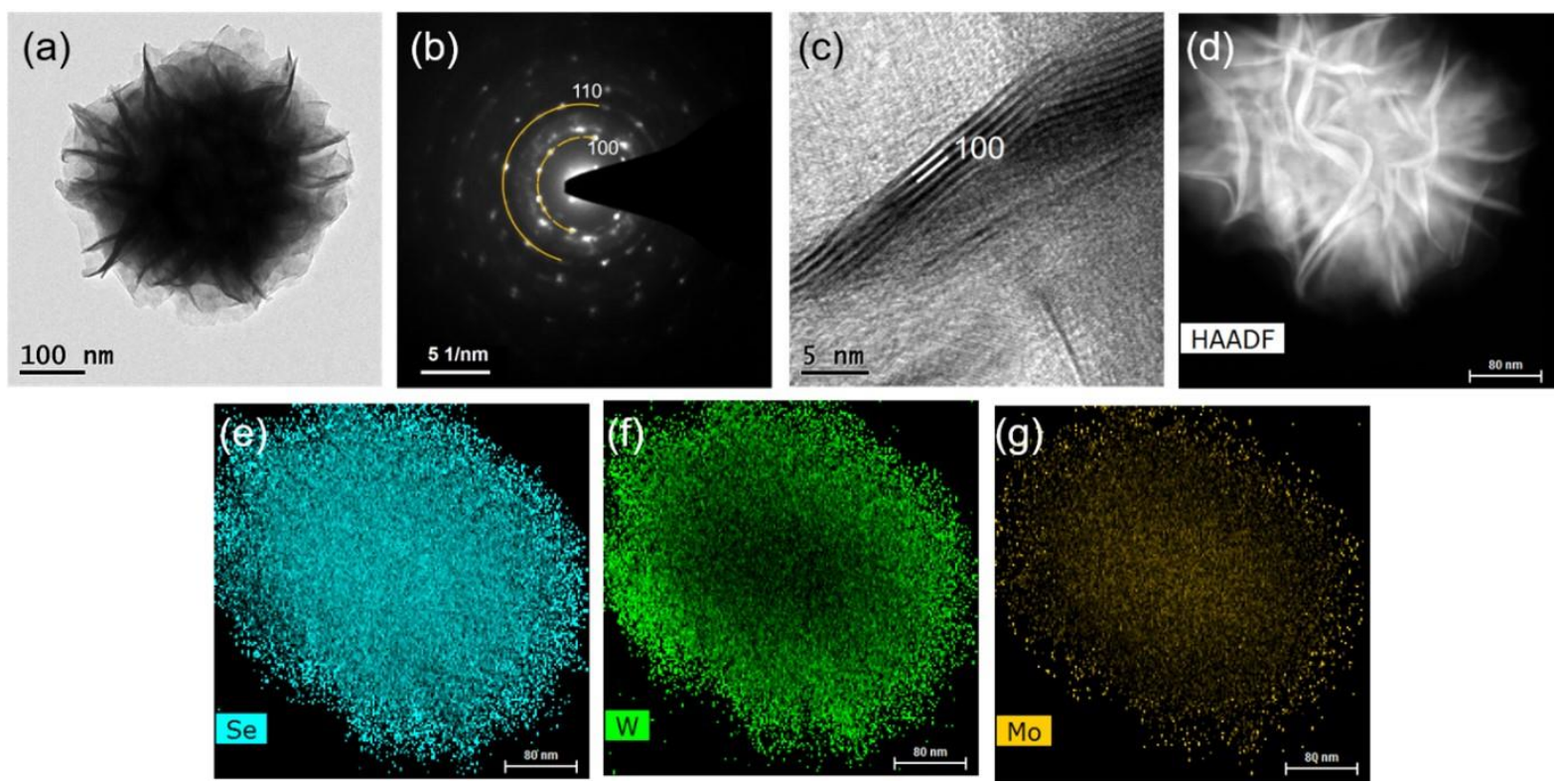

Figure 2. Bright-field TEM micrograph, Selected area electron diffraction pattern and high-resolution TEM of MoSe2@WSe2 (a, b and c) and corresponding HAADF-STEM image and elemental distribution of Se, W, Mo (d, $\mathrm{e}, \mathrm{f}$ and $\mathrm{g}$ ) respectively.

\section{References}

1. G. Fiori, F. Bonaccorso, G. Iannaccone, T. Palacios, D. Neu-maier, A. Seabaugh, S. K. Banerjee and L. Colombo, Naturenanotechnology, 2014,9, 768-779

2. C. Anichini, W. Czepa, D. Pakulski, A. Aliprandi, A. Ciesiel-ski and P. Samorì, Chemical Society Reviews, 2018,47, 4860-4908.

3. S. R. Kadam, A. N. Enyashin, L. Houben, R. Bar-Ziv and M. Bar-Sadan, Journal of Materials Chemistry A, 2020,8,1403-1416

4. Y. Zhao, C. Yang, G. Mao, J. Su, G. Cheng and W. Luo, Inorganic Chemistry Frontiers, 2018,5, 2978-2984.

5. Y. Yang, H. Fei, G. Ruan, C. Xiang and J. M. Tour, Advanced materials, 2014,26, 8163-8168

6. T. F. Jaramillo, K. P. Jørgensen, J. Bonde, J. H. Nielsen,S. Horch and I. Chorkendorff, science, 2007,317, $100-102$ 